\title{
SOME APPLICATIONS OF THE DIRICHLET INTEGRAL TO THE THEORY OF SURFACES
}

\author{
BY
}

L. C. YOUNG

1. The Dirichlet integral has been used by many writers to derive results in the theory of surfaces, and a number of these results are of fundamental importance. However, this theory is still far from satisfactory in certain respects: certainly if anything of the nature of compactness is to be obtained, the fundamental notions require considerable revision, for the Fréchet definition of a surface and of convergence cannot lead to this. On the other hand the work of Morrey shows how much the analytical character of a surface can be simplified by the simple operation of removing closed subsurfaces, an operation which does not fit naturally into the Fréchet scheme. The main object of this note is to study the effect of a similar operation, that of smoothing down approximately closed portions of surfaces, on the convergence of sequences of surfaces. In this work, the Dirichlet integral is again the principal tool of investigation. The proofs of our main results also make essential use of the notion of "intrinsic" area, recently introduced $[11]\left({ }^{1}\right)$.

Part of the paper deals with theorems of McShane and Morrey [5, 6], concerning equi-continuity and quasi-conformality: these are familiar enough, but we require certain minor extensions and corollaries. Our treatment of these partly follows an account of Morrey's results given by Cesari [3].

\section{Existence of A CERTAin GRATING}

2. A surface $S$ defined parametrically on the unit square $R$ of the plane of $w=u+i v$ by means of a continuous vector function $x=f(w)$ will be termed a Dirichlet surface $(N)$, and its parametric representation a Dirichlet representation $(N)$, if

(2.1)(i) $f$ is absolutely continuous on almost all parallels to the axes of $u$ and $v$;

(ii) $\iint_{R} 2^{-1}\left\{\left(f_{u}\right)^{2}+\left(f_{v}\right)^{2}\right\} d u d v<N$.

The left-hand side of (2.1) (i) will be termed the Dirichlet integral of $f$ in $R$. We denote further, for $0 \leqq t \leqq 1$, by $R^{\prime}(t)$ and $R^{\prime \prime}(t)$ the two rectangles consisting of the points $(u, v)$ of $R$ for which $u \leqq t$ and $v \leqq t$ respectively; and we define the "partial Dirichlet integral"

$$
I(t)=\iint_{R^{\prime}(t)} 2^{-1}\left(f_{v}\right)^{2} d u d v+\iint_{R^{\prime \prime}(t)} 2^{-1}\left(f_{u}\right)^{2} d u d v,
$$

Presented to the Society, February 28, 1948; received by the editors, July 25, 1947.

(1) Numbers in brackets refer to the references cited at the end of the paper. 
as a function of $t$, which increases for $0 \leqq t \leqq 1$ from 0 to $I(1)<N$. We denote by $T_{f}$ the set of $t$ such that $f(u, v)$ is absolutely continuous on the line $u=t$ and on the line $v=t$; by (2.1) (i), the set $T_{f}$ comprises almost all $t$ of the interval $0 \leqq t \leqq 1$

(2.2) In any interval $t^{\prime} \leqq t \leqq t^{\prime \prime}$ in which $I(t)$ has an incrementary ratio $\left[I\left(t^{\prime \prime}\right)-I\left(t^{\prime}\right)\right] /\left(t^{\prime \prime}-t^{\prime}\right) \leqq 2^{-1} \alpha^{2}$, there is a value $t=c$ of $T_{f}$ such that, for $0 \leqq u_{0} \leqq u_{0}+h \leqq 1$ and $0 \leqq v_{0} \leqq v_{0}+h \leqq 1$, we have
(i)
$\int_{u_{0}}^{u_{0}+h}\left|f_{u}(u, c)\right| d u \leqq \alpha \cdot h^{1 / 2}$ and
(ii) $\quad \int_{v_{0}}^{v_{0}+h}\left|f_{v}(c, v)\right| d v \leqq \alpha \cdot h^{1 / 2}$.

Proof. We have

$$
\frac{1}{t^{\prime \prime}-t^{\prime}} \int_{t^{\prime}}^{t^{\prime \prime}} g(t) d t \leqq \alpha^{2} \text { where } g(t)=\int_{0}^{1}\left[f_{u}(u, t)\right]^{2} d u+\int_{0}^{1}\left[f_{v}(t, v)\right]^{2} d v \text {. }
$$

Therefore there is a value $t=c$ of $T_{f}$ between $t^{\prime}$ and $t^{\prime \prime}$ for which $g(c) \leqq \alpha^{2}$. With this value of $c$, the left-hand side of (i) is, by Schwarz's inequality, at most

$$
h^{1 / 2} \cdot\left\{\int_{u_{0}}^{u_{0}+h}\left[f_{u}(u, c)\right]^{2} d u\right\}^{1 / 2} \leqq h^{1 / 2} \cdot\{g(c)\}^{1 / 2} \leqq h^{1 / 2} \cdot \alpha .
$$

Similarly we prove (ii).

3. The representation $x=f(w)$ will be said to possess a semi-elongated $(\epsilon, \delta)$-grating, if there exist a finite number of parallels to the coordinate axes, which subdivide $R$ into rectangles whose sides lie between $\delta$ and $2 \delta$, such that when $w$ describes any segment of length less than $\delta$ situated on the portion interior to $R$ of any one of these parallels, the length of the corresponding arc of curve described by $f(w)$ is less than $\epsilon$.

(3.1) There exists a positive number $\eta$ depending only on the positive numbers $N, \epsilon$ with the following property: if $x=f(w)$ is any Dirichlet representation $(N)$, then there is a number $\delta$ subject to $\eta<\delta<\epsilon$, such that $x=f(w)$ possesses a semi-elongated $(\epsilon, \delta)$-grating.

Proof. Suppose this false for the given value of $\epsilon$, and choose $\eta_{n}=1 /(3 n+2)$ : then there would exist, for each $n$, a surface with a Dirichlet representation $(N)$ of the form $x=f_{n}(w)$ which possesses no semi-elongated $(\epsilon, \delta)$-grating such that $\eta_{n}<\delta<\epsilon$. We shall show that this leads to a contradiction.

For this purpose, we select from the $f_{n}(w)$ an infinity of functions $f(w)$ such that the corresponding partial Dirichlet integrals $I(t)$ tend to a limitfunction. This is possible since the functions $I(t)$ are increasing, and we observe that the limit-function $i(t)$ itself increases for $0 \leqq t \leqq 1$ from $i(0)=0$ to $i(1) \leqq N$.

We denote by $E$ a finite set of $t$ such that the sum of the "jumps" $i(t+0)$ 
$-i(t-0)$ for all $t$ outside $E$ is less than $(\epsilon / 4)^{2}$. Since $i(t)$ is a sum of jumps together with a continuous increasing function, there is an integer $n^{\prime}$ such that

$$
i\left(t^{\prime \prime}\right)-i\left(t^{\prime}\right)<(\epsilon / 4)^{2}
$$

for every closed interval $t^{\prime} \leqq t \leqq t^{\prime \prime}$ of length less than $\eta_{n^{\prime}}$ containing no point of $E$. We may suppose further, by increasing the value of $n^{\prime}$ if necessary, that $\eta_{n^{\prime}}<\epsilon / 2$, and that if we divide the unit interval of $t$ into $3 n^{\prime}+2$ meshes of equal length $\eta_{n^{\prime}}$ no such mesh contains more than one point of $E$.

In every third mesh of such a subdivision (in increasing order of the meshes), we can determine an interval $t^{\prime} \leqq t \leqq t^{\prime \prime}$ of length $\eta_{n^{\prime}} / 4$ containing no point of $E$. In each of the $n^{\prime}$ intervals $t^{\prime} \leqq t \leqq t^{\prime \prime}$ so obtained, we may now apply (3.2), and we deduce that, for an infinity of our functions $I(t)$,

$$
I\left(t^{\prime \prime}\right)-I\left(t^{\prime}\right)<(\epsilon / 4)^{2} .
$$

Among the functions $I(t)$ satisfying (3.3) simultaneously for the $n^{\prime}$ intervals $t^{\prime} \leqq t \leqq t^{\prime \prime}$ concerned, we may choose one which corresponds to an $f(w)$ of the form $f_{n}(w)$ where $n \geqq n^{\prime}$; writing $\delta=2 \eta_{n^{\prime}}$, we then have $\eta_{n^{\prime}}<\delta<\epsilon$ and a fortiori $\eta_{n}<\delta<\epsilon$.

This function $I(t)$ has on each of the $n^{\prime}$ intervals $t^{\prime} \leqq t \leqq t^{\prime \prime}$ to which (3.3) applies an incrementary ratio less than $2^{-1} \epsilon^{2} / \delta$; hence by (2.2), we can determine in each of these intervals a value $t=c$ such that the arcs of curve described by $f(u, c)$ and by $f(c, v)$, as $u$ or $v$ describe a segment of length $\delta$, are of length less than $\epsilon$.

Since the differences of consecutive values of $c$ in increasing order clearly lie between $\delta$ and $2 \delta$, the representation $x=f(w)$ possesses a semi-elongated $(\epsilon, \delta)$-grating, defined by the $n^{\prime}$ pairs of lines $u=c$ and $v=c$.

Since $\eta_{n}<\delta<\epsilon$, this contradicts the definition of the function $f(w)=f_{n}(w)$, and so completes the proof.

\section{Equi-Continuity}

4. By the oscillation Osc $(f ; W)$ of a vector function $f(w)$ on a set $W$, we shall mean the diameter of the set of values $f(W)$.

In the sequel, we shall repeatedly deal with sequences of functions $f_{n}(w)$ which satisfy the following condition of smoothness:

(4.1) Given $\epsilon>0$, there exist $\delta>0$ and $n_{0}>0$ depending on $\epsilon$ only, so that the inequalities $n \geqq n_{0}$, Osc $\left(f_{n} ; W^{\prime}\right) \geqq \epsilon$, and Osc $\left(f_{n} ; W^{\prime \prime}\right) \geqq \epsilon$ together imply Osc $\left(f_{n} ; W\right) \geqq \delta$, in one or both the following situations:

(i) Whenever $W$ is an arc in $R$ whose extremities lie on the perimeter of $R$, and $W^{\prime}, W^{\prime \prime}$ are the two arcs of the perimeter of $R$ which are separated by the extremities of $W$;

(ii) Whenever $W$ is a simple closed curve in $R$ and $W^{\prime}, W^{\prime \prime}$ are the parts of $R$ respectively interior and exterior to $W$.

We observe that the conditions of smoothness (4.1) (i) and (4.1) (ii)-by 
which we mean the validity of (4.1) subject to (i), and its validity subject to (ii) - are independent of the representations selected for a sequence of continuous surfaces:

(4.2) Suppose that, for each $n$, the surface given by $x=f_{n}(w)$ has the further representation $x=g_{n}(w)$. Then if the sequence of functions $f_{n}(w)$ satisfies either of the conditions of smoothness, so does the sequence of the functions $g_{n}(w)$.

Proof. Denoting by $\phi_{n}(w)$ a function which defines a homeomorphism of $R$, the function $f_{n}\left[\phi_{n}(w)\right]$, which may clearly be substituted for $f_{n}(w)$ without affecting the validity of either condition of smoothness, can be made to differ by as little as we please from the function $g_{n}(w)$.

5. We say that a sequence of functions $f_{n}(w)$ satisfies on a set $E$ a threepoint condition, if $E$ contains for each $n$ three points $a_{n}, b_{n}, c_{n}$ whose mutual distance exceed some fixed $\delta>0$, such that the three values $f_{n}\left(a_{n}\right), f_{n}\left(b_{n}\right)$, $f_{n}\left(c_{n}\right)$ tend to three distinct limits as $n \rightarrow \infty$.

(5.1) Suppose that the vector equations $x=f_{n}(w)$ for $w$ in $R$ denote a sequence of Dirichlet representations $(N)$ and that the functions $f_{n}(w)$ satisfy a threepoint condition on the perimeter. Then these functions are equi-continuous

(i) on the perimeter, if they satisfy (4.1) (i);

(ii) in $R$, if they satisfy (4.1) (i) and (ii).

Proof. (i) For the $\epsilon$ in (4.1) (i) choose a sufficiently small number $\epsilon^{\prime}>0$ and use the three-point condition: then there exist $\delta^{\prime}, n^{\prime}$ so that if $n>n^{\prime}$ the relation Osc $\left(f_{n} ; W\right)<\delta^{\prime}$ implies Osc $\left(f_{n} ; W^{\prime}\right)<\epsilon^{\prime}$ whenever $W$ is an arc in $R$ of length less than $\delta^{\prime}$ joining two points of the perimeter, and $W^{\prime}$ is the smaller arc of the perimeter with the same extremities as $W$.

Choosing the number $\delta^{\prime} / 6$ for the $\epsilon$ of (3.1), we determine a corresponding $\eta$. For each $n>n^{\prime}$ the representation $x=f_{n}(w)$ then possesses a semi-elongated $(\epsilon, \delta)$-grating, where $\eta<\delta<\epsilon=\delta^{\prime} / 6$ : this grating divides the perimeter of $R$ into arcs $W^{\prime}$ of length greater than $\eta$, such that Osc $\left(f_{n} ; W^{\prime}\right)<\epsilon^{\prime}$, since the extremities of each $W^{\prime}$ can be joined on the grating by an arc $W$ of length less than $6 \delta$ for which Osc $\left(f_{n} ; W\right)<6 \epsilon=\delta^{\prime}$.

For $n \leqq n^{\prime}$ we can choose a number $\gamma<\eta$ such that the perimeter can be divided into equal arcs of length $\gamma$, on which the oscillation of each of the functions $f_{n}$, where $n \leqq n^{\prime}$, is less than $\epsilon^{\prime}$.

Clearly, whenever $\alpha, \beta$ are points of the perimeter distant less than $\gamma$, we now have for all $n$ without exception: $\left|f_{n}(\beta)-f_{n}(\alpha)\right|<2 \epsilon^{\prime}$, which proves (i).

(ii). Similarly, from (4.1) (ii) and the three-point condition, whenever $W^{\prime}$ is a sufficiently small rectangle in $R$ and $W$ is its perimeter, relations of the form $n>n^{\prime}$ and Osc $\left(f_{n} ; W\right)<\delta^{\prime}$ imply Osc $\left(f_{n} ; W^{\prime}\right)<\epsilon^{\prime}$. Moreover, by (3.1) and what has been proved in (i), there is an $\eta>0$, and for each $n>n^{\prime}$ a semielongated $(\epsilon, \delta)$-grating where $\eta<\delta<\epsilon$ and where $\epsilon$ is sufficiently small, which divides $R$ into rectangles $W^{\prime}$ on whose perimeters $W$ we have Osc $\left(f_{n} ; W\right)<\delta^{\prime}$; from this we derive Osc $\left(f_{n} ; W^{\prime}\right)<\epsilon^{\prime}$, and the argument proceeds as before. 
By very slight alterations of this proof, we could substitute in (ii) a three-point condition in $R$ for the three-point condition on the perimeter of $R$. Similarly, from (4.1)(ii) alone without three-point condition and without (4.1)(i), we could prove the $f_{n}$ equi-continuous in any square $R^{\prime}$ in the interior of $R$ such that $\operatorname{Lim}_{n}$ inf $\operatorname{Osc}\left(f_{n} ; R-R^{\prime}\right)>0$.

\section{Special cases: Theorems of McShane and Morrey}

6. The surface $x=f(w)$ will be said to have a split boundary-curve, if there exist on the perimeter of $R$ two complementary arcs $W^{\prime}, W^{\prime \prime}$, on neither of which $f$ has oscillation zero, whose extremities $w^{\prime}, w^{\prime \prime}$ are situated on a continuum of constancy of $f$.

The surface $x=f(w)$ will be said to have a closed subsurface, different from itself, if there exist a subdomain $D$ of $R$ on whose boundary $f$ is constant, such that neither of the quantities $\operatorname{Osc}(f ; D)$, Osc $(f ; R-D)$ vanishes. The closed subsurface in question is then defined by the vector equation $x=g(w)$, where $g$ is the function coinciding with $f$ in $D$ and on the boundary of $D$, and which is constant in $R-D$.

A surface which has neither a split boundary-curve nor a closed subsurface will be termed a basic surface (the term is substantially Whyburn's). We observe that a surface with a topological representation on $R$, or as we shall say a nondegenerate surface, is always a basic surface.

Finally we say that a sequence of surfaces has an asymptotic split boundary-curve $C$, if a subsequence $S_{n}$ of the surfaces has boundary-curves $C_{n}$ with the Fréchet limit $C$ such that the following situation arises: some double point of $C$ divides it into two closed subcurves (neither of which reduces to a point) which are expressible as Fréchet limits of two corresponding closed curves $C_{n}{ }^{\prime}, C_{r}{ }^{\prime \prime}$ on $S_{n}$, where $C_{n}{ }^{\prime}, C_{n}{ }^{\prime \prime}$ consist respectively of two complementary arcs of $C_{n}$ together with a same arc $K_{n}$ whose diameter tends to 0 .

We shall prove the following results:

(6.1) In order that the condition of smoothness (4.1) (i) be satisfied by a sequence of surfaces whose boundary-curves converge in the Frechet sense, it is necessary and sufficient that there be no asymptotic split boundary curve. (The condition is thus in particular satisfied if the boundary-curves of the surfaces tend to a simple closed curve, or again, to a point.)

(6.2) In order that the conditions of smoothness (4.1) (i) and/or (ii) be satisfied by a sequence of surfaces which tend in the Fréchet sense to a surface $S$, it is necessary and sufficient that $S$ have no split boundary-curve and/or no closed subsurface (different from itself).

The following theorems of McShane and Morrey, which we give in a slightly extended form, are immediate corollaries of (6.1) and (6.2) in view of (5.1).

(6.3) Suppose that the sequence of surfaces $S_{n}$ have no asymptotic split 
boundary-curve and that their boundary-curves converge. (This is in particular the case if their boundary-curves converge to a simple closed curve.) Suppose further that the surfaces have Dirichlet representations $(N)$ of the form $x=f_{n}(w)$ on $R$ which satisfy the three-point condition on the perimeter. Then the functions $f_{n}(w)$ are equi-continuous on the perimeter of $R[5$, p. 721, Lemma 2].

(6.4) Suppose that a basic surface $S$ is the limit of a sequence of surfaces, which possess Dirichlet representations $(N)$ of the form $x=f_{n}(w)$ on $R$ satisfying a three-point condition on the perimeter of $R$ (or, indeed, on $R$ itself). Then the functions $f_{n}$ are equi-continuous in $R$ ( $[6$, p. 699, Theorem I $]$; see also $[3$, p. 64, Theorem I]).

7. Preliminary reductions. Since a topological transformation of the perimeter of $R$ can always be completed to a topological transformation of $R$, we may suppose in (6.1) that the sequence of surfaces is given by the equations $x=f_{n}(w)$, where the functions $f_{n}(w)$ converge uniformly on the perimeter of $R$. We may suppose similarly in (6.2) that our surfaces have the form $x=f_{n}(w)$, where the $f_{n}$ converge uniformly in $R$. We remark further that:

(7.1) If (4.1) (i) or (ii) is satisfied by a sequence of functions $f_{n}(w)$, it is satisfied also by any sequence of functions $g_{n}(w)$ such that the difference $f_{n}-g_{n}$ tend uniformly to 0 .

From these facts it follows easily, by taking in (6.2) a sequence of repetitions for our $g_{n}$, while in the case of (6.1) we define $g_{n}$ so as to make the difference $f_{n}-g_{n}$ harmonic in $R$ with the boundary values $f_{n}-\lim f_{n}$, that it is enough to establish (6.2) in the case in which we have a sequence of repetitions of a single surface, and (6.1) in the case in which the given surfaces have a fixed boundary-curve.

We shall see that in the case of a sequence of functions $f_{n}(w)$ defined in $R$ which coincide on the perimeter of $R$ with a continuous function $f(w)$, the condition (4.1) (i) is equivalent to the following condition:

(7.2) The relations: $f\left(w^{\prime}\right)=f\left(w^{\prime \prime}\right)$, Osc $\left(f ; W^{\prime}\right) \neq 0$, Osc $\left(f ; W^{\prime \prime}\right) \neq 0$, together imply: $\operatorname{Lim} \inf _{n} \operatorname{Osc}\left(f_{n} ; W_{n}\right) \neq 0$, whenever the two points $w^{\prime}, w^{\prime \prime}$ are common extremities, of two complementary arcs $W^{\prime}, W^{\prime \prime}$ on the perimeter of $R$, and also of each of the arcs $W_{n}$ in $R$.

Again, in the case of a sequence of functions $f_{n}(w)$ consisting of repetitions of a same function $f(w)$ continuous in $R$, we shall see that (4.1) (i) and (4.1) (ii) are equivalent, respectively, to the conditions (7.3) (i) and (7.3) (ii) which follow:

(7.3) If $W$ is a subcontinuum of $R$, the relations: Osc $\left(f ; W^{\prime}\right) \neq 0$, Osc $\left(f ; W^{\prime \prime}\right) \neq 0$ together imply Osc $(f ; W) \neq 0$, whenever

(i) $W^{\prime}, W^{\prime \prime}$ are subsets of the perimeter of $R$ which are separated on this perimeter by points of $W$;

(ii) $W^{\prime}, W^{\prime \prime}$ are subsets of $R$ which are separated in the plane by $W$.

8. Proof of (6.1). In this proof we also establish:

(8.1) In the case in which the functions $f_{n}(w)$ defined in $R$ coincide on the 
perimeter of $R$ with a continuous $f(w),(4.1)$ (i) and (7.2) are equivalent.

Suppose (4.1) (i) satisfied, and also the first three relations in (7.2): then, by choosing $\epsilon$ less than the numbers Osc $\left(f ; W^{\prime}\right)$, Osc $\left(f, W^{\prime \prime}\right)$, we infer that Osc $\left(f_{n} ; W_{n}\right) \geqq \delta$ for all large $n$, so that (7.2) holds, and this clearly excludes the existence of an asymptotic split boundary-curve.

Conversely, suppose (4.1) (i) not satisfied for a certain $\epsilon$ : then there is a sequence $W_{n}$ of arcs in $R$ with extremities $w_{n}^{\prime}, w_{n}^{\prime \prime}$ on the perimeter, such that, for all large $n$ of a certain subsequence, in which Osc $\left(f_{n} ; W_{n}\right) \rightarrow 0$, we have: Osc $\left(f ; W_{n}^{\prime}\right) \geqq \epsilon$, Osc $\left(f ; W_{n}^{\prime \prime}\right) \geqq \epsilon$, where $W_{n}^{\prime}{ }^{\prime} W_{n}^{\prime \prime}$ are the two arcs into which $w_{n}^{\prime}, w_{n}^{\prime \prime}$ divide the perimeter of $R$. Moreover we may suppose that these two points tend to limits $w^{\prime}, w^{\prime \prime}$. By continuity of $f$ we may prolong $W_{n}$ to have the extremities $w^{\prime}, w^{\prime \prime}$ and modify correspondingly the complementary $\operatorname{arcs} W_{n}^{\prime}, W_{n}^{\prime \prime}$ which now become $W^{\prime}, W^{\prime \prime}$ independent of $n$, without affecting the above relations, except that $\epsilon / 2$ now replaces $\epsilon$. This implies that (7.2) cannot hold, and that there is an asymptotic split boundary-curve.

This completes the proof.

9. Proof of (6.2). We shall follow a simplified form of the argument of Cesari [3, p. 64, Theorem 1]. It is enough to prove:

(9.1) In the case in which the functions $f_{n}$ are repetitions of a same continuous function $f$, (4.1) (i) and (ii) are respectively equivalent to (7.3) (i) and (ii).

To establish this equivalence, we first recall the following well known facts:

(9.2) If for a sequence subcontinua $W_{n}$ of $R$ there is a point $w_{n}$ of $W_{n}$ which tends to a limit as $n \rightarrow \infty$, then the $W_{n}$ have a continuum of accumulation $W$, possibly reducing to a point. For a continuous $f(w)$ we then have $\operatorname{Lim}_{n}$ Osc $\left(f ; W_{n}\right)$ $=0$ if and only if Osc $(f ; W)=0$. Finally, if the interiors of two fixed circles are separated by each $W_{n}$, they are separated by $W$.

Of these statements, the first is a special case of Theorem (9.1), p. 14, of Whyburn's book Analytic topology, except that we use the terminology of Zoretti (to whom Whyburn refers at the end of the chapter). The second statement is obvious. Finally, if the third statement were false, we could join the circles by a polygon $L$ not meeting $W$, but meeting, for each $n, W_{n}$ in a point $a_{n}$; both $L$ and $W$ would contain a limit point $a$ of the points $a_{n}$, which is impossible since $L$ does not meet $W$.

We can now pass on to the proof of (9.1).

Suppose that (4.1) (i) does not hold. By (8.1) neither does (7.2); hence there exist arcs $W_{n}$ in $R$ for which $\operatorname{Lim}_{n} \operatorname{Osc}\left(f ; W_{n}\right)=0$ whose extremities $w^{\prime}$ and $w^{\prime \prime}$ are fixed points of the perimeter of $R$ with the following properties: at $w^{\prime}$ and $w^{\prime \prime}$ the function $f$ takes a same value $k$; and at two points $w=a$, $w=b$ separated by $w^{\prime}, w^{\prime \prime}$, on the perimeter, we have $f(w) \neq k$. Let $W$ be the continuum of accumulation of the $W_{n}$. By $(9.2), f(w)=k$ throughout $W$ since $W$ contains $w^{\prime}$ (and $\left.w^{\prime \prime}\right)$ : this implies that $a, b$ do not lie on $W$ and if we denote by $W^{\prime}$ and $W^{\prime \prime}$ the open arcs of the perimeter complementary to $W$, which contain $a$ and $b$ respectively, we find that (7.3) (i) does not hold. 
Conversely, if (7.3) (i) is not satisfied, then two points $w^{\prime}, w^{\prime \prime}$ of the perimeter are situated on a continuum $W$ in $R$ on which $f$ takes a constant value $k$, and these two points separate two further points $w=a$ and $w=b$ of the perimeter, at which $f(w)$ differs from $k$ by at least $2 \epsilon>0$. We can join $w^{\prime}$, $w^{\prime \prime}$ in $R$ by a polygon whose vertices lie on $W$ and whose sides are arbitrarily small. Since the oscillation of $f$ on such a polygon is as small as we please (in view of the fact that $f$ is constant at the vertices), it follows easily that (4.1) (i) cannot hold.

Again, suppose that (4.1) (ii) does not hold. Then for a certain $\epsilon>0$, there exist for each $n$ : a simple closed curve $W_{n}$ in $R$, a point $w_{n}$ of $W_{n}$ and two points $w_{n}^{\prime}, w_{n}^{\prime \prime}$ of $R$ separated in the plane by $W_{n}$, such that

$$
\operatorname{Osc}\left(f ; W_{n}\right)<1 / n,\left|f\left(w_{n}{ }^{\prime}\right)-f\left(w_{n}\right)\right| \geqq \epsilon / 2 \text { and }\left|f\left(w_{n}{ }^{\prime \prime}\right)-f\left(w_{n}\right)\right| \geqq \epsilon / 2 \text {. }
$$

We may suppose further, by restricting ourselves to a subsequence, that $w_{n}, w_{n}^{\prime}, w_{n}^{\prime \prime}$ tend to limits $c, c^{\prime}, c^{\prime \prime}$. Consider two circular discs with centres at $c^{\prime}, c^{\prime \prime}$, in which $f$ has oscillation less than $\epsilon / 4$; neither disc can contain a point of $W_{n}$ for large $n$, since otherwise $\epsilon / 2<\epsilon / 4+\operatorname{Osc}\left(f ; W_{n}\right)<\epsilon / 4+1 / n$. Hence the discs are separated by $W_{n}$ for all large $n$, and therefore by the continuum of accumulation $W$ of the $W_{n}$ in view of (9.2). Furthermore by (9.2), $f$ has on $W$ the constant value $f(c)$ which differs (by at least $\epsilon / 2$ ) from both $f\left(c^{\prime}\right)$ and $f\left(c^{\prime \prime}\right)$. Hence taking $W^{\prime}, W^{\prime \prime}$ to be the open segments joining $c^{\prime}, c^{\prime \prime}$ respectively to the nearest points of $W$, we find that (7.3) (ii) is not satisfied.

Finally, if (7.3) (ii) is not satisfied, there is a continuum $W$ in $R$, on which $f$ takes a constant value $k$, which separates two points of $R$ at which $|f-k|$ $>2 \epsilon$. We cover $W$ by a finite number of squares with sides parallel to the axes, situated in $R$, in each of which the oscillation of $f$ is less than a preassigned $\delta>0$. The boundary of this sum of squares is then a sum of polygons separating our two points, and it contains a simple closed curve with the same property. On this curve the oscillation of $f$ is less than $\delta$, and its values there all differ by at least $2 \epsilon-\delta$ from its values at the two given points which are separated by the curve. This shows that (4.1) (ii) cannot hold.

Both the equivalences asserted are thus established.

\section{MoRREy's THEOREM AND ONE OF ITS CONSEQUENCES}

10. For a fixed surface, we shall term "minimal" a Dirichlet representation $x=f(w)$ for which the Dirichlet integral of $f(w)$ is a minimum. We shall term "quasi-conformal" a Dirichlet representation for which the Dirichlet integral coincides with the classical area.

(10.1) A basic surface of finite Lebesgue-Fréchet area, whose boundary-curve does not reduce to a point, has a quasi-conformal representation.

It is of importance to us that this statement of Morrey's theorem [6, p. 701, Theorem 2] is not restricted to surfaces situated in three-dimensional 
space - a remark which will enable us to derive the following corollary:

(10.2) The classical area of a Dirichlet surface coincides with the minimum, whether attained or not, of its Dirichlet integral. In particular, a minimal Dirichlet representation is quasi-conformal.

The corollary may also be expressed in the form: "A Dirichlet surface whose classical area is less than $N$ is a Dirichlet surface $(N)$."

To deduce this from (10.1), let $x=f(w)$ be a Dirichlet representation of the given surface, and suppose, by adding to the dimensionality of the underlying space if necessary, that there exist two coordinate axes at right angles to one another and to those of a certain linear subspace containing the values of $f(w)$. We denote by $g(w)$ the vector whose components along these two fresh axes are the coordinates $u, v$ of $w$, and whose remaining components vanish.

The nondegenerate Dirichlet surface $S_{\epsilon}$ represented by $x=f(w)+\epsilon \cdot g(w)$ is a basic surface. A simple inequality of the type $(a+b+c)^{1 / 2} \leqq a^{1 / 2}+b^{1 / 2}+c^{1 / 2}$ shows that its classical area exceeds that of the original surface $S$ at most by the arbitrarily small amount: $\epsilon+\epsilon^{1 / 2} \cdot \iint_{R}\left\{\left(f_{u}\right)^{2}+\left(f_{v}\right)^{2}\right\}^{1 / 2} d u d v$, where the double integral converges with the Dirichlet integral of $f$. Hence by choice of $\epsilon$, the Lebesgue-Fréchet area of $S_{\epsilon}$, which coincides with its classical area, is less than $N$.

But (10.1) then implies that $S_{\epsilon}$ has a Dirichlet representation $x=F(w)$ such that the Dirichlet integral is less than $N$, and this Dirichlet integral is decreased by replacing by 0 the components of $F(w)$ along the two additional axes. To complete the deduction of (10.2) from (10.1), it is enough to verify that this operation turns the representation $x=F(w)$ of $S_{\epsilon}$ into a corresponding Dirichlet representation of the original surface, that is, to produce a homeomorphism $\phi(w)$ of $R$ for which the relevant projection of $F[\phi(w)]$ differs arbitrarily little from $f(w)$ : we do so by observing that the difference in question is less than that of the functions $F[\phi(w)]$ and $f(w)+\epsilon \cdot g(w)$, which is as small as we please since $F(w)$ defines a representation of $S_{\epsilon}$.

It should be noted that the above establishes (10.2) for polyhedra from the corresponding special case of (10.1), which reduces to a classical result of Schwarz (see [7, p. 16]).

11. As regards (10.1) itself, the following arrangement of the argument, beginning with a few lemmas, seems to us to bring out most clearly the essential simplicity of the version of Morrey's proof, based on (6.4), which is given by Cesari ( $[3$, p. 83 , Theorem II $]$ which the reader will have no difficulty in identifying with our (10.1), in spite of an unfortunate misprint):

(11.1) The uniform limit of a Dirichlet representation $(N)$ of a surface $S_{n}$ is a Dirichlet representation of the surface lim $S_{n}$ for which the Dirichlet integral does not exceed the corresponding lower limit of Dirichlet integrals.

This is proved, for instance, by McShane [5, pp. 719-721, Lemma 1].

(11.2) If $x=f(w)$ is a Dirichlet representation, and $\phi(w)$ defines a Lipschitzian, or again a conformal, transformation of $R$ into itself, or part of itself, 
then $x=f[\phi(w)]$ is a Dirichlet representation of the same or of a different surface, and the partial derivatives of $f[\phi(w)]$ are given, almost everywhere in $R$, by the usual formulae for composite derivation.

The first part is elementary when $f$ is Lipschitzian, and the general case follows at once by (11.1) if we approximate to $f$ by a Lipschitizian function with a bounded Dirichlet integral. The second part follows from the fact that partial derivatives are almost everywhere the coefficients of an approximate differential [9, p. 300, Theorem 12.2].

(11.3) A minimal Dirichlet representation of a polyhedron is quasi-conformal.

To avoid appealing to the classical result of Schwarz, Cesari [3, pp. 77-83] gives a variational proof which, apart from the implicit use of (11.2) is purely elementary. This proof makes no essential use of the assumption of an underlying three-dimensional space.

We now come to the main part of Cesari's proof. In what follows, the three-point condition can be dealt with trivially, on account of (11.2), by means of a conformal transformation of $R$ into itself, the boundary-curve of each of the surfaces concerned being assumed not to reduce to a point.

This being so we deduce easily from (6.4) and (11.1) that:

(11.4) $A$ basic Dirichlet surface, whose boundary-curve does not reduce to a point, possesses a minimal Dirichlet representation.

In particular, by (11.3), this establishes (10.1) and so (10.2), in the case of a polyhedron. Now a surface $S$ of finite Lebesgue-Fréchet area $A$ is the limit of a polyhedron $S_{n}$ of area less than $A+\epsilon$, and by what has just been proved, $S_{n}$ has a Dirichlet representation $x=f_{n}(w)$ for which the Dirichlet integral is less than $A+2 \epsilon$. In the case of a basic Dirichlet surface, whose boundarycurve does not reduce to a point, it now follows easily from (6.4) and (11.1) that $S$ has a Dirichlet representation for which the Dirichlet integral is at most $A+2 \epsilon$. Since the Dirichlet integral is clearly never less than $A$, we deduce that for the minimal Dirichlet representation in (11.4), the Dirichlet integral equals $A$, that is, this representation is quasi-conformal.

This completes the proof of (10.1).

\section{LEMMAS BASED ON HARMONIC INTERPOLATION}

12. We shall have occasion, several times, to use the following result:

(12.1) If $x=f(w)$ is a Dirichlet representation $(N)$, and $D$ denotes a simplyconnected subdomain of $R$ on each of whose prime ends $f(w)$ is constant, then a further Dirichlet representation ( $N)$, of the same or of a different surface, is defined by the vector equation $x=f^{*}(w)$, where $f^{*}(w)$ is the continuous function in $R$ which is harmonic in $D$ and which coincides with $f(w)$ outside $D$.

The function $f^{*}(w)$ will be termed in $D$ the harmonic interpolation of $f(w)$; its existence is proved, for instance, in [11, between (7.3) and (7.4)]. In order to prove (12.1), it is sufficient to show that: 
(12.2) The Dirichlet integral is not increased by harmonic interpolation.

This is proved for instance by McShane (On the minimizing property of the harmonic function, Bull. Amer. Math. Soc. vol. 40 (1934) p. 594, Theorem 1).

13. As a first application of harmonic interpolation, we prove:

(13.1) Let $x=f(w)$ define a surface of finite Lebesgue-Fréchet area $A$, which does not possess a split boundary-curve. Then there exists a basic Dirichlet surface $x=g(w)$ of Lebesgue-Fréchet area not greater than $A$, such that $g(w)=f(w)$ except in a sum of simply-connected disjoint domains interior to $R$ in each of which $g(w)$ is constant.

We first prove that the Lebesgue-Fréchet area is not increased when we pass from a surface $x=f(w)$ to a surface $x=g(w)$, where $g(w)$ coincides with $f(w)$ except in a sum of simply-connected disjoint subdomains $D$ in each of which $g(w)$ is constant. For this purpose, we express the surface $x=f(w)$ as the limit of polyhedra whose areas tend to its Lebesgue-Fréchet area $A$. We denote by $x=f_{n}(w)$ a Dirichlet representation of one of these polyhedra, whose area is less than $A+1 / n$ and whose distance from $x=f(w)$ is less than $1 / n$. By $(10.2)$ we can choose the representation $x=f_{n}(w)$ so that the Dirichlet integral of $f_{n}$ is less than $A+1 / n$.

Denoting by $\phi_{n}(w)$ a homeomorphism of $R$ such that $\left|f_{n}\left[\phi_{n}(w)\right]-f(w)\right|$ $<1 / n$, we cover the boundary of the open set consisting of the sum of the domains $\phi_{n}(D)$ by a finite number of squares, with sides parallel to the axes, in each of which the oscillation of the function $f_{n}(w)$ is less than $1 / n$. If we remove these squares from the open set $\sum \phi_{n}(D)$ there will remain a finite number of polygonal domains $G$. It is easy to see that each $G$ is interior to one of the domains $\phi_{n}(D)$ and hence that it is simply-connected.

We now define a function $g_{n}(w)$ equal to $f_{n}(w)$ outside the domains $G$ and to its harmonic interpolation in each $G$. Clearly the Dirichlet integral of $g_{n}$ is less than $A+1 / n$, and therefore the area of the surface $x=g_{n}(w)$ is less than $A+1 / n$.

Furthermore we find that $\left|g_{n}\left[\phi_{n}(w)\right]-g(w)\right|<2 / n$. Making $n \rightarrow \infty$, this shows that the surface $x=g(w)$ is the limit of the surface $x=g_{n}(w)$ of area less than $A+1 / n$, so that by lower-semicontinuity of the Lebesgue-Fréchet area, the surface $x=g(w)$ has Lebesgue-Fréchet area not exceeding $A$, as asserted above.

14. We are now in a position to establish (13.1). We may clearly suppose that the boundary-curve of the given surface $x=f(w)$ does not reduce to a point. This being so, we associate with each point $w$ of $R$ a maximal subdomain of $R$ containing $w$ and on whose boundary $f$ is constant, whenever the point $w$ is separated from the perimeter of $R$ by at least one continuum of constancy.

We denote by $g(w)$ the function equal to $f(w)$ outside every such a maximal domain, and constant in the closures of such domains. By what has 
been proved, the surface $x=g(w)$ has a finite Lebesgue-Fréchet area not greater than $A$. To complete the proof of (13.1), it is thus sufficient, in view of (10.1), to show that this surface is basic.

Suppose it is not. Then there exist at least two subdomains $W^{\prime}, W^{\prime \prime}$ of $R$, on neither of which $g$ is constant, separated in $R$ by a maximal subcontinuum $W$ of $R$ on which $g$ has a constant value $c$. We observe that $f=c$ at every boundary point of the set $W$, and so throughout the continuum $K$ which bounds the infinite domain $G$ complementary to $W$.

By its definition, the function $g$ is thus constant in $R-G$, since $f$ is constant on its boundary $K$. Hence $G$ contains $W^{\prime}$ and $W^{\prime \prime}$; this implies that $W^{\prime}$ and $W^{\prime \prime}$ are separated in $R$ by $K$ on which $f=c$ and that each of $W^{\prime}, W^{\prime \prime}$ has on its boundary an arc of the perimeter of $R$ on which $f \neq c$. This is incompatible with (7.3) (i), and so contradicts our assumptions.

15. We pass on to an application which concerns the notion of area.

So far, in this note, we have used the Lebesgue-Fréchet area, which of course agrees with the classical area as given by the usual double integral inthe case of a Dirichlet representation. For this fact, which we have used without special comment, reference may be made, for instance to $[7$, p. 13].

However, in the sequel, we shall make essential use of two quite different notions: the Banach area, which depends on the representation, and the intrinsic area which depends only on the surface. These notions are defined in [11].

The reader must be careful to distinguish these various interpretations of area: Besicovitch [2] shows that there exists a surface homeomorphic with a disc, which has finite Lebesgue-Frechet area, but infinite intrinsic and Banach areas. This surface is a Dirichlet surface, in fact, by (10.1), it has a quasiconformal representation.

This example shows in particular that a Dirichlet surface $(N)$ may have an intrinsic area greater than $N$, and even infinite. We know $[11,(9.1)]$ that:

(15.1) The intrinsic area of a Dirichlet surface is never less than its classical area.

To supplement this result, we now establish the following application of (12.1), which goes some way towards remedying the state of affairs:

(15.2) Suppose that the vector equation $x=f(w)$ is a Dirichlet representation $(N)$ of a surface $S$ whose classical area is $A$. Then for any $\alpha>0$, where $A+\alpha<N$, there exists a surface $S^{*}$, whose Banach area on the interior $R^{0}$ of $R$ is less than $A+\alpha$, and which is given by a Dirichlet representation $(N)$ of the form $x=f^{*}(w)$ subject to the following conditions:

The function $f^{*}(w)$ coincides with $f(w)$ outside a sequence of rectangles situated in the interior of $R$ and of arbitrarily small total plane measure;

The sum of the areas (in any sense) of the pieces of $S^{*}$ which correspond to these rectangles is less than $\alpha$.

Moreover, if the boundary-curve of $S$ has intrinsic area $a<\infty$, the intrinsic 
area of $S^{*}$ is less than $A+a+\alpha$.

In the proof, we shall make use of a lemma which is implicitly contained in a proof of a theorem given by Saks $[9$, p. 301]:

(15.3) In order that a finite function $f(w)$ which is measurable on a plane set $Q$ be almost everywhere on $Q$ approximately derivable with respect to each of the variables $u$ and $v$ (where $w=u+i v$ ), it is necessary and sufficient that almost all points of $Q$ be contained in the sum of a sequence of measurable sets $R_{n}$ in each of which $f$ is Lipschitzian.

This is proved explicitly in $[11,(9.3)]$ : it will be observed that in the case in which $f$ is bounded in $Q$, the sets $R_{n}$ there constructed form an expanding sequence.

Proof of (15.2). From this last remark, coupled with (15.3) itself, it follows from the hypotheses of (15.2) that there exists an open subset $E$ of $R^{0}$, of arbitrarily small plane measure, such that $f$ is Lipschitzian in $R^{0}-E$ and has a Dirichlet integral over $E$ not exceeding $\alpha$.

By (3.1) there exist everywhere dense sets of parallels to the axes in $R$ on which $f(w)$ describes rectifiable curves. We can therefore express $E$ as the sum of a sequence of closed rectangles, with only boundary-points in common, such that the boundary-curves described by $f(w)$ on their perimeters are rectifiable. We denote by $G$ the complementary domains situated in these rectangles, of the whole sets consisting of the elements meeting these perimeters. We define $f^{*}$ to be the function which coincides with $f$ outside the domains $G$, and with the harmonic interpolation of $f$ in each of these domains.

On the domains $G$, the surface $S^{*}$ defined by the vector equation $x=f^{*}(w)$ has a Banach area which agrees with the corresponding classical area, and which therefore cannot exceed the Dirichlet integral of $f^{*}$ on $\sum G$, and a fortiori that of $f$ on $E$. The Banach area of the part of $S^{*}$ on $\sum G$ is thus at most $\alpha$. The Banach area of the part of $S^{*}$ on $E-\sum G$ vanishes, since this part of $S^{*}$ is situated on an enumerable number of rectifiable curves.

Thus by addition, the Banach area of $S^{*}$ on $E$ is not greater than $\alpha$.

On the other hand, on $R^{0}-E$ the functions $f$ and $f^{*}$ agree and are Lipschitzian: hence on $R^{0}-E$ the classical areas of $S$ and $S^{*}$ agree and [11, Theorem 4.3] coincide with the corresponding Banach areas. Thus the Banach area of $S^{*}$ on $R^{0}-E$ is certainly less than $A$.

By addition, the Banach area of $S^{*}$ on $R^{0}$ is less than $A+\alpha$.

Finally, if the boundary-curve of $S$, which coincides with that of $S^{*}$, has intrinsic area $a<\infty$, it follows at once from the definitions $\left({ }^{2}\right)$ of the Banach multiplicity-functions and of the Morrey multiplicity-functions, which are associated with $S^{*}$ as image of $R$, or again with its boundary-curve as image of

(2) We use the principle, worth noting for its own sake, that the Morrey multiplicity function corresponding to the sum of two sets $E_{1}+E_{2}$ of the parameter domain cannot exceed the sum of the Morrey multiplicity function corresponding to $E_{1}$ and the Banach multiplicity corresponding to $E_{2}$. 
the perimeter of $R$, that the intrinsic area of $S^{*}$ on $R$ is less than $A+a+\alpha$.

This completes the proof.

\section{SOME CONSEQUENCES OF "A LEMMA IN THE THEORY OF SURFACES"}

16. We make use of the following generalization [11, Theorem 8.2] of "a lemma in the theory of surfaces" [10]:

(16.1) There is an absolute constant $K$ such that any surface $S$ of finite intrinsic area $A$ whose boundary lies in a sphere of radius a can be so modified as to lie in a sphere of radius $b=a \cdot \exp (K / \epsilon)$, the modification being made by replacing its parametric representation $f(w)$ by its harmonic interpolation in a finite number of simply-connected subdomains of the fundamental rectangle $R$, in such a manner that the total area of these harmonic portions of the new surface is less than $\epsilon \cdot A$ and that their boundaries lie on a finite number of rectifiable curves situated on $S$.

The final assertion is not made in the result quoted, but is clearly established in the proof. It implies that the intrinsic area of the part of the surface $S$, or of its modification, which corresponds to elements meeting the bound. aries of the subdomains in question is zero. The reader should note that an alteration of $f(w)$ in a subdomain $G$ affects the surface in two ways: by shifting points of the surface which correspond to points of $G$ and by altering the structure of elements (continua of constancy of $f$ ) which may protrude outside $G$.

17. The lemmas on which our main theorems will be based will be derived from (16.1) and (3.1). We shall be concerned with functions $f(w)$ such that:

(i) The equation $x=f(w)$ defines a Dirichlet representation $(N)$;

(ii) The surface so represented has finite intrinsic area $A(f)$;

(iii) On the perimeter of $R$ we have $\left|f\left(w^{\prime}\right)-f\left(w^{\prime \prime}\right)\right|<\omega(\rho), \omega(\rho)$ a certain fixed function of the distance $\rho$ of the points $w^{\prime}, w^{\prime \prime}$, and $\omega(\rho) \rightarrow 0$ as $\rho \rightarrow 0$.

The validity of (iii) is equivalent to equi-continuity on the perimeter, but the explicit function $\omega=\omega(\rho)$ will appear in our statements.

18. We begin with the following result:

(18.1) Given $\epsilon>0$, there is a number $\delta$ and a finite sequence of disjoint simplyconnected subdomains $G$ of $R$ associated with $f$, such that:

(i) $\eta<\delta<\epsilon$, where $\eta=\eta(N, \omega)>0$ is independent of $f$;

(ii) The diameter of each $G$ is less than $\delta$;

(iii) $f$ is constant on each prime end of $G$;

moreover, denoting by $f^{*}$ the function equal to $f$ in $R-\sum G$ and to the harmonic interpolation of $f$ in each $G$, by $\Gamma$ the sum of elements in $R$ for the surface $x=f^{*}(w)$ (that is, the sum of those continua of constancy of $f^{*}$ ) which meet $\sum G$, and by $\gamma$ the sum of those which meet the boundaries of the $G$ :

(iv) Osc $\left(f^{*}, a\right)<\epsilon$ on any segment $s$ in $R$ of length less than $\delta$ :

(v) the part of the surface $x=f^{*}(w)$ represented on $\Gamma$ has intrinsic area less 
than $\epsilon \cdot A(f)$ (so that in particular the intrinsic area of the surface $x=f^{*}(w)$ on $R$ is less than $(1+\epsilon) \cdot A(f))$;

(vi) The set $f(\gamma)$, which coincides with $f^{*}(\gamma)$, lies on a finite number of rectifiable curves.

Proof. Since the function $\omega(\rho)$ of the preceding paragraph (the modulus of continuity on the perimeter) is fixed, we can use (3.1) to determine for each positive $a$ an $\eta>0$ independent of $f$, and at the same time for each $f$ a semielongated grating which divides $R$ into rectangles $R_{k}$ whose sides lie between $\delta / 4$ and $\delta / 2$, where $\eta<\delta<a$, so that the oscillation of $f$ on the perimeter of each $R_{k}$ is less than $a$.

We shall choose $a=(\epsilon / 16) \cdot \exp (-K / \epsilon)$ and we apply (16.1) with $b=\epsilon / 16$ and with $R_{k}$ in place of $R$.

In each $R_{k}$ we modify the function $f$ by harmonic interpolation in a finite number of disjoint simply-connected subdomains $G$. The modified function, which we denote by $f^{*}(w)$, is by addition defined throughout $R$.

The first three assertions of (18.1) are clearly satisfied: (i), because $a<\epsilon$; (ii), because each $G$ lies in an $R_{k}$ of side less than $\delta / 2$; and (iii), as this is implied by our definition of harmonic interpolation.

Moreover (iv) is satisfied, because by (16.1) the oscillation of $f^{*}$ in each $R_{k}$ is less than $2 b=\epsilon / 8$, and because those $R_{k}$ which meet a same $1 / 4$ of $s$ must have a common point since this $1 / 4$ can only penetrate into at most two adjacent strips of the grating parallel to an axis.

Again, if we denote by $A_{k}$ the intrinsic area of the portion of surface defined by $x=f(w)$ on $R_{k}$, and by $\Gamma_{k}, \gamma_{k}$ the sums of those elements in $R_{k}$ for the modified portion of surface $x=f^{*}(w)$, which meet the sum of the $G$ in $R_{k}$, or which meet their boundaries, respectively, then (16.1) implies that the intrinsic area of the part of the surface $x=f^{*}(w)$ on $\Gamma_{k}$ is not greater than $\epsilon \cdot A_{k}$ and that the set $f\left(\gamma_{k}\right)$, which coincides with $f^{*}\left(\gamma_{k}\right)$, lies on a finite number of rectifiable curves. The assertions ( $\mathrm{v}$ ) and ( $\mathrm{vi}$ ) follow at once by addition, if we observe that the elements of the two surfaces $x=f(w)$ and $x=f^{*}(w)$ which meet the grating correspond to values of $x$ situated on a finite number of rectifiable curves, and so contribute nothing to the intrinsic area.

19. The operation of modifying $f$ in (18.1) will be denoted by $H(\epsilon)$ so that we shall write the resulting function $f^{*}=H(\epsilon) \cdot f$. Similarly, when $f$ has undergone a first operation $H\left(\epsilon_{1}\right)$, we may subject the resulting function to a second operation $H\left(\epsilon_{2}\right)$, since the function $H\left(\epsilon_{1}\right) \cdot f$ satisfies the same conditions: (i), (ii), (iii) of $\$ 17$, as the function $f$, the intrinsic area $A\left(H\left(\epsilon_{1}\right) \cdot f\right)$ being less than $\left(1+\epsilon_{1}\right) \cdot A(f)$.

Given positive numbers $\epsilon_{1}, \epsilon_{2}, \cdots$, we write for the left-hand product of the $m$ first modifications:

$$
g_{m}(w)=\left\{\prod_{k \leqq m} H\left(\epsilon_{k}\right)\right\} \cdot f=H\left(\epsilon_{m}\right) \cdots \cdot H\left(\epsilon_{1}\right) \cdot f=\left\{\prod_{r \leqq k \leqq m} H\left(\epsilon_{k}\right)\right\} \cdot g_{r} .
$$


In particular $g_{0}(w)$ is the function $f(w)$.

Further, when we use (18.1) to pass from $g_{r-1}$ to $g_{r}$, instead of from $f$ to $f^{*}$, the relevant symbols $\eta, \delta, G$ will be affected by a suffix $r$. Moreover we write $E_{r}$ for the sum of all the (possibly overlapping) subdomains $G_{k}$ of the various stages for which $k \leqq r$; we note that $g_{r}$ need not be harmonic in the (possibly multiply-connected) constituent regions of $E_{r}$.

However:

(19.1) The function $g_{r}(w)$ is "piecewise harmonic" in $E_{r}$, by which we mean that $E_{r}$ is the sum of an open set $O_{r}$ in each of whose constituent regions the function is harmonic, together with the set $E_{r}-O_{r}$ in which the values of the function are situated on a finite number of rectifiable curves. We easily verify this state of affairs, as a consequence of (18.1) (vi).

Moreover, denoting by $W_{r}$ the whole set obtained by adding to $E_{r}$ those elements in $R$ for the surface $x=f(w)$ which meet the boundary of $E_{r}$ (or, what amounts to the same since $f(w)=g_{r}(w)$ outside $E_{r}$, the corresponding elements for the surface $x=g_{r}(w)$, so that $W_{r}$ is a whole set for both surfaces), we see similarly that the set $f\left(W_{r}-E_{r}\right)$ which coincides with $g_{r}\left(W_{r}-E_{r}\right)$ is situated on a finite number of rectifiable curves.

These remarks joined to (18.1) (v) imply:

(19.2) The classical area on $E_{r}$ and the intrinsic area on $W_{r}$ coincide for the surface $x=g_{r}(w)$, and do not exceed the value

$$
\left[\left\{\prod_{k \leqq r}\left(1+\epsilon_{k}\right)\right\}-1\right] \cdot B .
$$

Again, (18.1) (iv) implies that Osc $\left(g_{r}, s\right)<\epsilon_{r}$ for a segment $s$ of length less than $\delta_{r}$. However the oscillation of a subsequent $g_{m}$, where $m>r$, on the segment $s$ may of course be greater than this. We shall prove that:

(19.3) If $m \geqq r$ and the segment $s$ has length less than $\delta_{r}$, then

$$
\text { Osc }\left(g_{m}, s\right)<\epsilon_{r}+\sum_{r<k \leqq m} 2 \cdot \epsilon_{k}<2 \cdot \sum_{r \leqq k \leqq m} \epsilon_{k} .
$$

It is clearly enough to prove that, for $k>r$,

(19.4) Osc $\left(g_{k}, s\right) \leqq \operatorname{Osc}\left(g_{k-1}, s\right)+2 \epsilon_{k}$.

To see this we may clearly suppose $s$ to have length not less than $\delta_{k}$, so that its ends lie in different subdomains $G_{k}$ or else one at least of them is a point at which the functions $g_{k}$ and $g_{k-1}$ coincide. In either case, $s$ contains two points at which these functions agree, at a distance less than $\delta_{k}$ from the two ends (the two points may possibly coincide, or one or both may be ends). It follows at once that the right-hand side of (19.4) is not less than the difference of the values of $g_{k}$ at the two ends of $s$. Applying this result to subsegments of $s$, we derive (19.4) at once, and so establish (19.3).

20. Consider now the case of an enumerable set of functions $f=f_{n}$ subject to the conditions (i), (ii), (iii) of $\$ 17$. We choose the numbers $\epsilon_{r}>0$ so that 
$\coprod\left(1+\epsilon_{r}\right)<1+\epsilon$, where $\epsilon$ is a preassigned positive number. This implies that

$$
\lambda_{r}=2 \cdot \sum_{k \geqq r} \epsilon_{k} \rightarrow 0 \quad \text { as } \quad r \rightarrow \infty .
$$

To each of our functions $f=f_{n}$, there now corresponds a sequence of modifications $g_{r}=g_{n, r}$. We denote by $\bar{f}_{n}$ the function $g_{n, n}$.

We see at once that the functions $\bar{f}_{n}$ are equi-continuous in $R$.

In fact, given $\alpha>0$, we can choose an integer $r$ and a positive number $\beta$ so that $\alpha>\lambda_{r}, \beta<\eta_{r}$, so as to ensure that for $n \leqq r$

(20.1) $\mid \bar{f}_{n}\left(w^{\prime}\right)-\bar{f}_{n}\left(w^{\prime \prime}\right)<\alpha$ when the points $w^{\prime}, w^{\prime \prime}$ of $R$ are distant less than $\beta$. But (20.1) then holds also for $n>r$ on account of (19.3), since the segment $s$ which joins $w^{\prime}$, $w^{\prime \prime}$ has length less than $\beta<\eta_{r}$ which is less than the number $\delta_{r}$ associated with the function $g_{n, r}$.

Combining this with results already established, we have:

(20.2) Suppose given an enumerable number of Dirichlet representations $(N)$ $x=f(w)$ of surfaces of finite intrinsic area $A(f)$, where the functions $f(w)$ are equi-continuous on the perimeter of $R$. Then given $\epsilon>0$, there exists for each $f$ a modification $\bar{f}=\bar{f}(w)$, such that the functions $\bar{f}$ are equicontinuous in $R$, the modification of each $f$ consisting in altering $f$ only in an open set $E$ in whose constituent regions $\bar{f}$ is piecewise harmonic (see (19.1) for the definition), in such a manner that the total area of these piecewise harmonic portions of each of the modified surfaces is less than $\epsilon \cdot A(f)$ and that their boundaries lie on a finite number of rectifiable curves. Furthermore the new equations $x=\bar{f}(w)$ are themselves Dirichlet representations $(N)$ of surfaces of finite intrinsic area $A(\bar{f})$ $<(1+\epsilon) \cdot A(f)$.

\section{QUASI-COMPACTNESS THEOREMS}

21. A set of surfaces will be termed "quasi-compact" if, given $\epsilon>0$, it can be transformed into a compact set of surfaces by the following process: certain portions of each surface, corresponding to open subdomains of the parameter domain, are replaced by new portions whose total area is less than $\epsilon$.

In this definition, the term "area" may be interpreted in various ways, but we shall in fact suppose the new portions sufficiently smooth to ensure that this makes no essential difference. We observe that the number of portions affected may be finite or enumerable: we have not thought it worth while to distinguish the two cases. Each portion corresponds to a constituent region of an open set in the parameter domain, so that there may be enumerably many such portions, and they need not be simply-connected.

22. As an immediate consequence of (20.2), we have firstly:

(22.1) A sequence of Dirichlet surfaces whose intrinsic areas are bounded and whose boundary-curves tend to a closed curve which is no asymptotic split boundary-curve, or in particular tend to a simple closed curve, is quasi-compact. 
The theorem remains valid if the limit of the boundary-curves reduces to a point, but we shall exclude this case which can be dealt with by a simplified form of our argument. This being so, the surfaces possess, by (15.1), (10.2), (11.2), for any number $N$ which exceeds the upper bound of their intrinsic areas, Dirichlet representations $(N)$ satisfying the three-point condition on the perimeter of $R$. By (6.3) we have equi-continuity on the perimeter, and our conclusion now follows from (20.2) since surfaces with equi-continuous representations and convergent boundary-curves constitute a compact set.

23. It is remarkable that the theorem just proved remains valid when we substitute in its statement classical areas for intrinsic areas. The result is then, by (15.1), a greatly strengthened theorem which asserts that:

(23.1) A sequence of Dirichlet surfaces whose classical areas are bounded and whose boundary-curves tend to a closed curve which is no asymptotic split boundary-curve (\$6) is quasi-compact.

Proof. Arguing as before, we see that the surfaces possess, for any number $N$ which exceeds the upper bound of their classical areas, Dirichlet representations $(N)$ of the form $x=f(w)$ equi-continuous on the perimeter of $R$.

We modify each $f$ in accordance with (15.2), and denote as there the resulting function by $f^{*}$. Let $\Delta_{\theta}=\Delta_{\theta}\left(f^{*}\right)$ be the upper bound of the difference $\left|f^{*}(t w)-f^{*}(w)\right|$ for $\theta \leqq t \leqq 1$. Since each $f^{*}$ is continuous, we can choose $\theta>1 / 2$ depending on $f^{*}$ and tending to 1 with sufficient rapidity from below, to ensure that $\Delta_{\theta}\left(f^{*}\right) \rightarrow 0$ as $f^{*}$ describes the sequence of modified functions.

Writing $F(w)=f^{*}(\theta w)$ for $w$ in $R$, we see that it follows from (15.2) that the surfaces $x=F(w)$ have intrinsic area (and indeed Banach area) less than $N$. We denote by $\bar{F}$ the modification of each $F$ in accordance with (20.2), whose requirements are now satisfied by the sequence of representations $x=F(w)$, and we define a further sequence of functions $\bar{f}$ by writing

$$
\bar{f}(w)=\bar{F}(w / \theta) \text { when }|u| \leqq \theta,|v| \leqq \theta, \quad \bar{f}(w)=f^{*}(w) \text { otherwise. }
$$

Since the functions $\bar{F}$ are equi-continuous in $R$, we easily verify that the same is true of the functions $\bar{f}$. Moreover we find that each $f$ coincides with the corresponding $\bar{f}$ outside an open subset of $R$ on which the classical area of $x=\bar{f}(w)$ is less than $\epsilon N+\alpha$, where $\epsilon, \alpha$ are at our disposal.

This completes the proof.

24. We now combine the result just proved with (13.1) and we observe that if the function $f$, in the statement of (13.1), is made to describe a sequence, and the corresponding surfaces $x=f(w)$ have no asymptotic split boundary-curve, then neither have the basic surfaces $\lambda=g(w)$ derived from them. We thus obtain, at the cost of strengthening the hypotheses concerning the boundary-curves, a theorem valid for surfaces of finite LebesgueFréchet area which need not be Dirichlet surfaces:

(24.1) A sequence of surfaces whose Lebesgue-Fréchet areas are bounded and whose boundary-curves are unsplit and tend to a closed curve which is no asymp- 
totic split boundary-curve (or, in particular, with simple boundary-curves tending to a simple closed curve) is quasi-compact.

\section{REFERENCES}

(The list of references should be supplemented from [8]; some of the papers cited below are not quoted in the final version of this note, but the reader should consult them in connection with any alternative lines of argument that may occur to him.)

1. A. S. Besicovitch, $A$ general form of the covering principle and relative differentiation of additive functions, Proc. Cambridge Philos. Soc. vol. 41 (1945) pp. 103-110; vol. 42 (1945) pp. $1-10$.

2. - On the definition and value of the area of a surface, Quart. J. Math. Oxford Ser. vol. 16 (1945) pp. 86-102.

3. L. Cesari, Criteri di uguale continuita ed applicazioni alla quadratura delle superficie, Ann. École Norm. (2) vol. 12 (1943) pp. 61-84.

4. H. Lebesgue, Sur le problème de Diric'let, Rend. Circ. Mat. Palermo vol. 24 (1907) pp. 371-402.

5. E. J. McShane, Parametrization of saddle surfaces with application to the problem of Plateau, Trans. Amer. Math. Soc. vol. 35 (1932) pp. 716-733.

6. C. B. Morrey, An analytic characterization of surfaces of finite Lebesgue area, Amer. J. Math. vol. 57 (1935) pp. 692-702.

7. T. Rad6, On the problem of Plateau, Ergebnisse der Mathematik, vol. 2, Berlin, Springer, 1933.

8. - Length and area, Amer. Math. Soc. Colloquium Publications, vol. 30, New York, 1948.

9. S. Saks, Theory of the integral, Monografje Mathematyczne, vol. 7, Warsaw, 1937.

10. L. C. Young, A lemma in the theory of surfaces, J. London Math. Soc. vol. 19 (1944) pp. 209-212.

11. - On area and length, to appear in Fund. Math.

University of CAPE Town,

Cape Town, South Africa 